\title{
Local Quantization Code Histogram for Texture Classification
}

\author{
Yang Zhao, Rong-Gang Wang, Wen-Min Wang, Wen Gao \\ School of Electronic and Computer Engineering, Peking University Shenzhen Graduate School, Shenzhen 518055, China
}

\begin{abstract}
In this paper, an efficient local operator, namely the Local Quantization Code (LQC), is proposed for texture classification. The conventional local binary pattern can be regarded as a special local quantization method with two levels, 0 and 1 . Some variants of the LBP demonstrate that increasing the local quantization level can enhance the local discriminative capability. Hence, we present a simple and unified framework to validate the performance of different local quantization levels. In the proposed LQC, pixels located in different quantization levels are separately counted and the average local gray value difference is adopted to set a series of quantization thresholds. Extensive experiments are carried out on several challenging texture databases. The experimental results demonstrate the LQC with appropriate local quantization level can effectively characterize the local gray-level distribution.
\end{abstract}

Key Words: Local binary pattern, Texture classification, Local quantization.

\section{Introduction}

Texture classification is a basic issue in image processing and computer vision, and playing a significant role in many applications, such as remote sensing, biomedical image analysis, image recognition and retrieval. In these practical applications, it is very difficult to ensure that captured images have the same viewpoint. Hence, texture classification methods should be ideally invariant to translation, rotation and scaling. 
More and more attention has been paid on invariant texture classification. So far, many approaches have been proposed to achieve rotation invariance for texture classification that can be broadly divided into two categories, i.e., statistical methods and model-based methods, respectively. In statistical methods, texture is generally described by the statistics of selected features, e.g., invariant histogram, texture elements, and microstructures. Davis et al. [1] exploited polarograms and generalized co-occurrence matrices to obtain rotation invariant statistical features. Duvernoy et al. [2] introduced Fourier descriptors to extract the rotation invariant texture feature on the spectrum domain. Goyal et al. [3] proposed a method by using texel property histogram. Eichmann et al. [4] presented texture descriptors based on line structures extracted by Hough transform. In [34], Hanbay et al. presented four effective rotation invariant features based on histograms of oriented gradients (HOG) and co-occurrence HOG (CoHOG). In model-based methods, texture is usually presented as a probability model or as a linear combination of a set of basis functions. Kashyap et al. [5] developed a circular simultaneous autoregressive (CSAR) model for rotation invariant texture classification. Cohen et al. [6] characterized texture as Gaussian Markov random fields and used the maximum likelihood to estimate rotation angles. Chen and Kundu [7] addressed rotation invariant by using multichannel sub-bands decomposition and hidden Markov model (HMM). Porter et al. [8] exploited the wavelet transform for rotation invariant texture classification by means of the Daubechies four-tap wavelet filter coefficients. Recently, Xu et al. [31]-[33] proposed a scale invariant texture feature by means of the multi-fractal spectrum.

McLean [15] proposed to use vector quantization for texture classification. But the quantization step is processed on large image area and thus loses the details of the local 
neighborhood grayscale distribution. However, the local distribution has been proven to be the important discriminative information of texture. For example, Haralick proposed that central to virtually all aspects of texture classification is the identification of a "texture cell" that defines a local region containing the essence of the repeated structure [16]; Effective texton-based methods [17],[28]-[30] also proved that texture classification can be tackled effectively by employing only local neighborhood distributions .

Local texture descriptor is another example to prove the importance of the local neighborhood distribution. In [9], Ojala et al. proposed an efficient local operator, namely Local Binary Pattern (LBP). The LBP extracts the local pattern and it is proven to be invariant to monotonic grayscale transformation. Nowadays, the LBP is one of the most popular local texture descriptors, since it is simple and effective [27]. Many LBP-like local texture operators have been proposed after Ojala's work, e.g., Heikkila et al. [10] proposed center-symmetric LBP (CS-LBP) by comparing center-symmetric pairs of pixels instead of comparing neighbors with central pixels. Liao et al. [11] presented Dominant LBP (DLBP), in which dominant patterns were experimentally chosen from all rotation invariant patterns. Tan and Triggs [12] proposed the method of Local Ternary Pattern (LTP), which extends original LBP to 3-valued codes. Recently, Guo et al. [13] developed the completed LBP (CLBP) by combining the conventional LBP with the measures of local intensity difference and central gray level. Khellah [14] presented a new method for texture classification, which combines Dominant Neighborhood Structure (DNS) and traditional LBP, Zhao et al. [18] proposed to use local binary count (LBC) to extract the local neighborhood distribution, Zhang et al. [19] presented a new local energy pattern for texture classification, Li et al. [20] proposed a scale 
invariant LBP by means of scale-adaptive texton. In addition, there is other way to look at LBP, e.g., LBP is regarded as a special filter-based method $[25,26]$.

The LBP can be regarded as a special local quantization method with two quantization levels, 0 and 1 . How to select the quantization level is a basic issue of the traditional quantization methods. The specified gray value of each individual pixel is sensitive to noise and illumination, thus lower quantization level is more robust to the illumination changes. But reducing the quantization level also loses detailed gray value information of pixel at the same time. The LBP is insensitive to monotonic illumination changes by quantizing the local gray level into only two levels. Meanwhile, the two values ( 0 and 1$)$ extract scarcely any gray value information of the pixel. Although the LBP discards almost all the gray value information of individual pixel, the quantized neighbor pixels are combined together to describe the local pattern. Therefore, the LBP can effectively characterize the local distribution. Is the local quantization level needed to be increased? Does an optimal local quantization level exist? How to describe the local distribution when the quantization level is increased? In this paper, we shall try to address these questions by proposing a new local operator named Local Quantization Code (LQC). Experimental results illustrate that the LQC with appropriate local quantization level can effectively characterize the local neighborhood distribution.

The rest of this paper is organized as follows: Section II briefly reviews the basic principle of the relative variants of the LBP. Section III presents the LQC in detail. Experimental results are presented in Section IV, and Section V concludes the paper.

\section{Related works and analyses}


In this Section we provided a brief review of the LBP and related variants of the LBP, i.e., the LTP, the CLBP and the LBC.

As shown in Fig.1, the algorithm of LBP contains three main steps. First, the values of neighbor pixels are turned into binary values ( 0 or 1$)$ by comparing them with the central pixel. Second, the binary numbers are encoded to characterize a local structure pattern, and then the code is transformed into decimal number. Finally, after the LBP code of each pixel is defined, a histogram will be built to represent the texture image.

\begin{tabular}{|c|c|c|c|c|c|c|c|c|}
\hline 24 & 82 & 78 & \multirow{3}{*}{$\underset{56}{\stackrel{\text { threshold }}{\longrightarrow}}$} & 0 & 1 & 1 & \multirow{2}{*}{ encoding } & \multirow{2}{*}{$\begin{array}{l}\text { Binary: } \\
01100110\end{array}$} \\
\hline 52 & 56 & 51 & & 0 & & 0 & & \\
\hline 56 & 66 & 55 & & 1 & 1 & 0 & & $\begin{array}{l}\text { Dec1ma1: } \\
102\end{array}$ \\
\hline
\end{tabular}

Fig.1. Illustration of the LBP process. $(\mathrm{P}=8, \mathrm{R}=1)$

Usually, the LBP encoding strategy can be described as follows:

$$
L B P_{P, R}=\sum_{p=0}^{P-1} s\left(g_{p}-g_{c}\right) 2^{p}, \quad s(x)=\left\{\begin{array}{l}
1, x \geq 0 \\
0, x<0
\end{array}\right.
$$

where $g_{c}$ represents the gray value of the center pixel and $g_{p}(p=0, \ldots, P-1)$ denotes the gray value of the neighbor pixel on a circle of radius $R$, and $P$ is the total number of the neighbors. As aforementioned, although the LBP is robust to monotonous illumination changes, the binary quantization process also loses the detailed gray value information of pixels. Hence, it seems that increasing the local quantization level can enhance the discriminative capability of the LBP. However, it is very difficult to compute the LBP codes directly when the local quantization level is increased. It is easy to be found that the length of LBP feature become $L^{p}$ if the quantization level increases to $L$, e.g., if local quantization level is 4 and 16 neighbors are calculated, the length of LBP-like feature will be $4^{16}$. 
Although it is hard to increase the local quantization level directly in the LBP-like way, many works have been proposed to extract the gray value information that omitted in the binary quantization step of the LBP.

Tan and Triggs [12] proposed local ternary pattern (LTP) to quantize the local neighbors into three levels. As illustrated in Fig. 2, 2-valued $(0,1)$ LBP code is extended to 3-valued (-1, $0,1)$ ternary code by means of a threshold $t$. The upper pattern and lower pattern are then encoded in LBP-like way, respectively. LTP codes can extract more gray value difference information, but no longer strictly invariant to monotonic gray scale transformation since threshold $t$ is specified by user. It also should be noticed that the threshold $t$ is set as 5 on many texture databases according to the experimental performance. Since the gray value of the pixels can be 0 to 255 , the local quantization threshold seems quite small, and this will also be discussed later in Section 3.2.

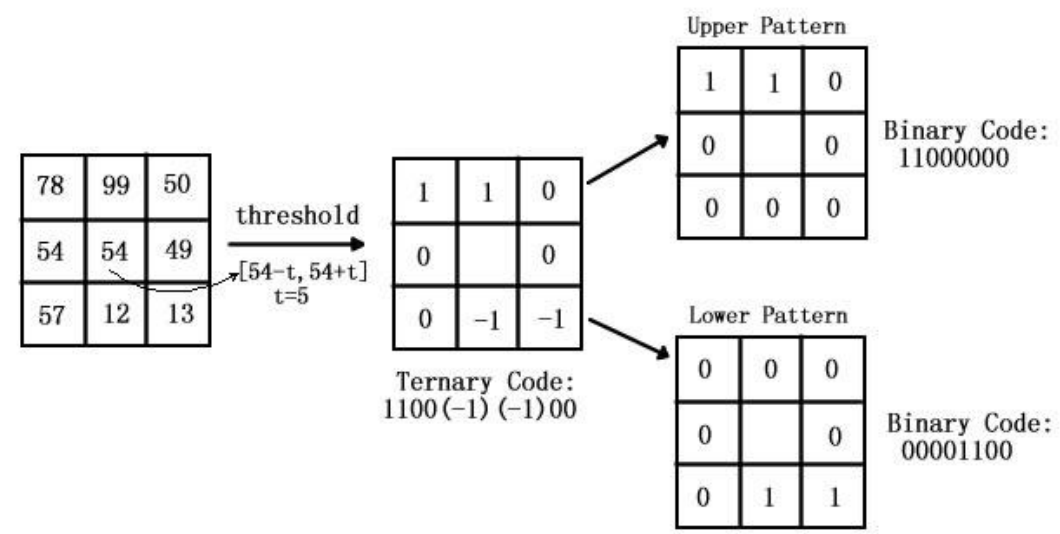

Fig.2. Illustration of the $\operatorname{LTP}(\mathrm{P}=8, \mathrm{R}=1)$.

Guo et al. [13] proposed a completed framework of LBP (CLBP) by combining the sign (0 or 1) feature with the magnitude (the gray value difference) feature. Although the CLBP did not directly increase the local quantization level, the magnitudes feature provided complementary gray value difference information that lost during the binary quantization 
process. Moreover, Guo et al. observed that the center pixel also had discriminative information. The CLBP extended original LBP to a completed framework and achieved impressive classification results.

These variants of the LBP demonstrate that increasing the local quantization levels can enhance the discriminative capability. Then the key question becomes how to increase the local quantization level in an efficient and unified framework.

In [18], Zhao et al. proposed the local binary count (LBC) by means of a local counting method to encode the rotation invariant local distribution after local neighbors are quantized into two levels. In the LBC, the number of value 1's in the binary neighbor sets is simply counted. As illustrated in Fig.3, the number of value 1's is 4 in the binary neighbor set, thus the LBC code of the central pixel is 4 . The LBC reveals another cursory encoding method to characterize the local neighborhood distribution, and the LBC-like encoding is easy to expand.

\begin{tabular}{|c|c|c|c|c|c|c|}
\hline 24 & 82 & 78 & \multirow{3}{*}{$\underset{56}{\stackrel{\text { threshold }}{\longrightarrow}}$} & 0 & 1 & 1 \\
\hline 52 & 56 & 51 & & 0 & & 0 \\
\hline 56 & 66 & 55 & & 1 & 1 & 0 \\
\hline
\end{tabular}

LBC Code: 4

Fig. 3. The illustration of the $\mathrm{LBC}(\mathrm{P}=8, \mathrm{R}=1)$

\section{Local quantization code histogram method}

\subsection{Calculation of the local quantization code}

In the conventional LBP and its variants, each pixel in the local neighbor set is turned into 
binary form by comparing it with the central pixel. To increase the quantization level, a series of quantization thresholds $\left(\sigma_{1}, \sigma_{2}, \sigma_{3}, \sigma_{4} \ldots\right)$ need to be used. After these thresholds are fixed, the pixels on the local neighbor set are classified into different quantization levels. The number of neighbor pixels located at the $i$-th quantization level $\left(q_{i}\right)$ is then counted as follows,

$$
q_{i}=\sum_{p=1}^{P} L\left(g_{p}-g_{c}\right), \quad L(x)=\left\{\begin{array}{cc}
1, & \text { if } \sigma_{i} \leq|x| \leq \sigma_{i+1} \\
0, & \text { else }
\end{array}\right.
$$

where $g_{c}$ represents the gray value of the center pixel and $g_{p}(p=1, \ldots, P)$ denotes the gray value of the neighbor pixel on a circle neighbor set, and $\sigma_{i}$ is the $i$-th quantization threshold. As in [9], the neighbors that do not fall in the center of pixels are estimated by bilinear interpolation. Obviously, computing the $q_{i}$ is equal to count the number of the neighbor pixels that are quantized into the $i$-th level.

After the $q_{i}$ at each local quantization level is calculated, we can define the Local Quantization Code (LQC) as follows:

$$
L Q C=\sum_{i=1}^{Q} q_{i} \times 10^{i-1}
$$

where $q_{i}$ is the number of neighbor pixels at quantization level $i$, and $Q$ represents the total number of the neighbor quantization levels.

The center pixel, which expresses the local gray level, also has discriminative information. Therefore the proposed LQC is also combined with the global quantization level of the central pixel:

$$
L Q C=q_{c} \times 10^{i}+\sum_{i=1}^{Q} q_{i} \times 10^{i-1}
$$

where $q_{c}$ represents the quantization level of the central pixel. In this paper, the central pixel is quantized by equally splitting the gray level histogram. The proposed LQC encoding scheme 
adopts a decimal system coding rather than commonly used binary system coding, thus the central quantization level $q_{c}$ can be set from 0 to 9 . Different to the neighbor pixels, the gray value of central pixel is quantized by means of global quantization method instead of local quantization. That is because $q_{c}$ is supposed to describe the level of local gray value among the whole image.

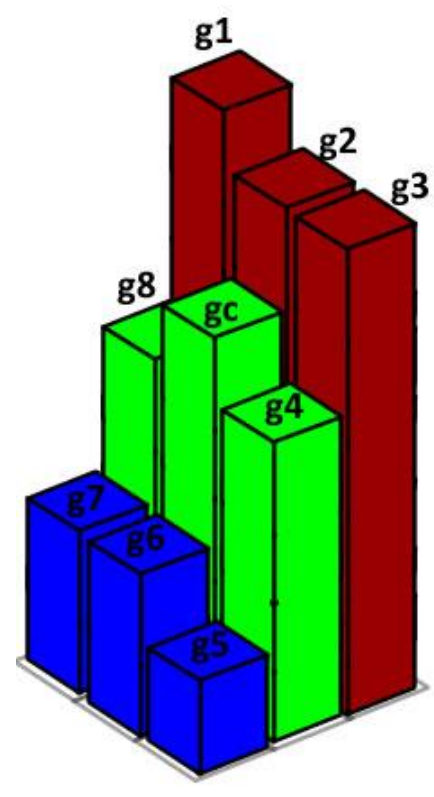

LBP code:00000111

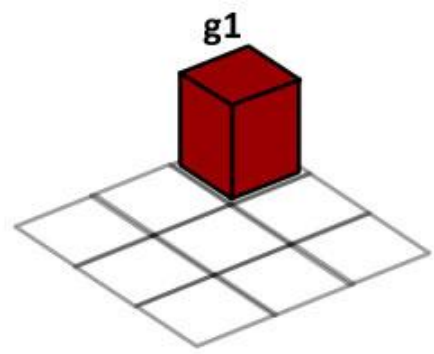

Level $4 \quad q_{4}=1$

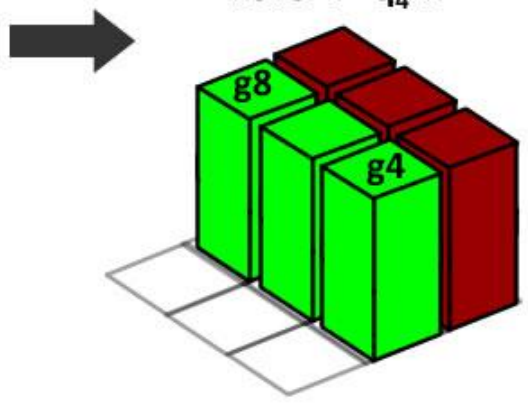

Level $2 \quad q_{2}=2$

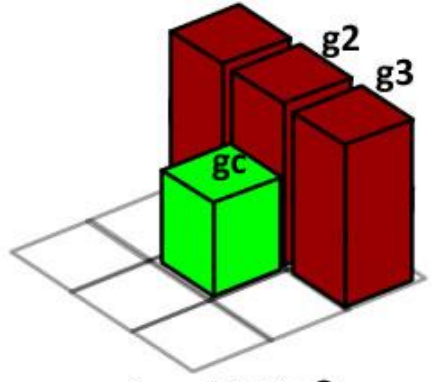

Level $3 q_{3}=2$

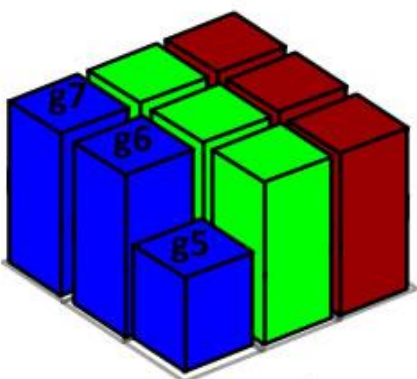

Level $1 \quad q_{1}=3$

LQC : $122(1,2,2,3)$

Fig. 4. Illustration of $\mathrm{LQC}$ at $(\mathrm{R}=1, \mathrm{P}=8)$.

Fig. 4 illustrates the encoding process of the $\mathrm{LQC}$ at $(\mathrm{R}=1, \mathrm{P}=8)$. Each cubic column denotes a pixel and the height of the cubic column represents the gray value of corresponding pixel. In Fig.4, gray value of neighbor pixels is quantized into 4 levels. Then the number $q_{i}$ $(i=1, \ldots, 4)$ of neighbor pixels located at each level is counted. There are 1, 2, 2, 3 pixels located at $1^{\text {st }}, 2^{\text {nd }}, 3^{\text {th }}$, and $4^{\text {th }}$ level, respectively. Finally, numbers of pixels at different levels are combined to form the LQC code 122 . We omit the last number 3 at $1^{\text {st }}$ level to reduce the feature size, since the total number of neighbor pixels is fixed $(1+2+2+3=8)$.

It is obvious that the LQC can accurately characterize the local structure by means of more 
quantization levels than traditional LBP. As shown in Fig. 5, different local structures may have the same LBP code. But their LQC codes are quite different from each other. Note that the LQC merely counting the number of pixels at different levels and thus is strictly invariant to rotation. As a result, the LQC encoding is very suitable for the rotation invariant texture classification scenario.

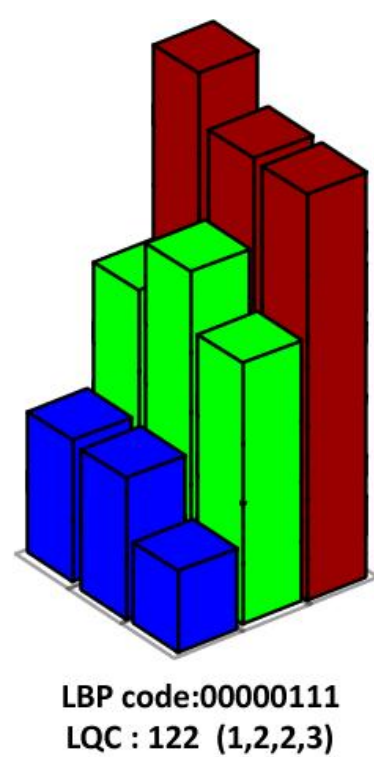

(a)

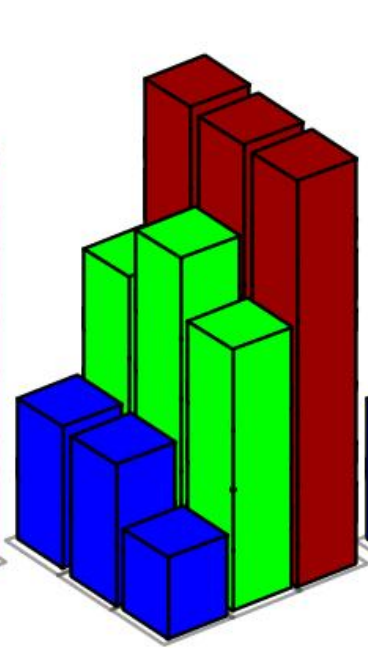

LBP code:00000111

LQC : $32(0,3,2,3)$

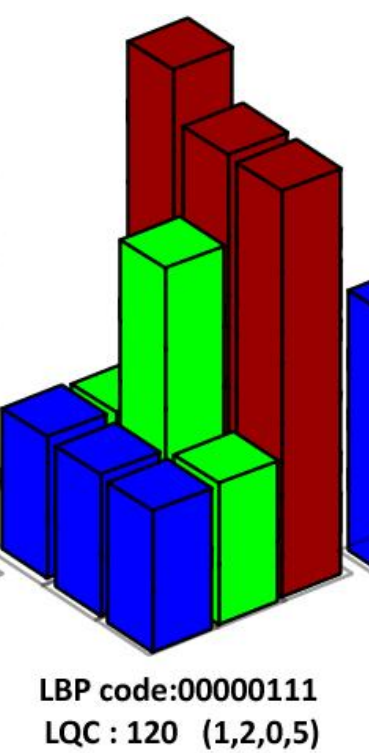

(c)

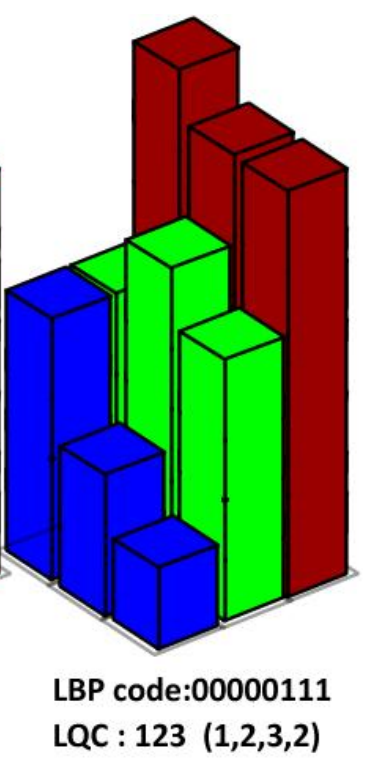

(d)

Fig. 5. The LQC can discriminate different local structures with same LBP code.

In this paper, the neighbor number $P$ at radius 1 is set as 8 , i.e., $(R=1, P=8)$, therefore the value of each $q_{i}$ can be nine value (0-8). When the radius of the neighbor set enlarged, the value of each $q_{i}$ is still quantized into nine values (0-8) so that the decimal system based Eqn. (4) can be used at larger radiuses. For example, the value range of $q_{i}$ is 0 to 16 when the radius is 2 , and this $q_{i}$ is then directly quantized into nine levels (0-8).

After the local quantization code of each pixel is calculated, a histogram is built to represent the texture image. It should be noted that although the size of LQC histogram is $10^{L}$ with quantization level $L$, many bins of the histogram are always zeros. That is because there are only finite neighbor pixels to be encoded. For example, if the $q_{4}$ at the quantization level 4 
is 8 with $(R=1, P=8)$, the LQC code only can be ' 800 ' since there are total 8 neighbor pixels. The rest codes ' 801 ' to ' 899 ' are thus insignificant and we can simply remove these meaningless all-zeros-bins from the final histogram. After removing the all-zeros-bins, the sizes of LQC histograms are 9, 45, 165, 495 with $\left(q_{c}=0, R=1, P=8\right)$ for quantization level of 2 ,

\section{$3,4,5$, respectively.}

In conventional LBP, the gray value of local central pixel is used as local threshold directly. It is clear that the fixed artificial threshold is not robust to discontinuous and non-uniform illumination transformations, while the local difference is relatively more insensitive to illumination transformations. Hence, we adopt the average local difference to set the series of quantization thresholds instead of fixed artificial thresholds. A base-threshold $\Delta$ is computed as the average difference of local neighbors on the texture image as follows:

$$
\Delta=\frac{1}{P \times N_{c}} \sum_{i=1}^{N_{c}} \sum_{p=1}^{P}\left|g_{p_{i}}-g_{c_{i}}\right|
$$

where $g_{p}(p=1, \ldots, P)$ and $g_{c}$ are defined as in Eqn. (1), $N_{c}$ is the total number of the central pixels on the texture image. The $\Delta$ reflects the average difference of local gray-level, which is more robust to monotonic illumination transformations than the artificial fixed threshold. In this paper, the series of quantization thresholds $\left(\sigma_{1}, \sigma_{2}, \sigma_{3}, \sigma_{4} \ldots\right)$ are simply set as $(0, \Delta, 2 \Delta$, $3 \Delta \ldots$ ) by means of the base-threshold $\Delta$ in this paper.

\subsection{The discussion of the quantization level}

The main question of the LQC is how to select the optimal quantization level. From Eqn. (3) we can find that the length of the feature vector becomes much longer when the local quantization level increases. In this paper, a LBC-like feature is used to characterize the local 
distribution instead of the LBP-like encoding. That is because the length of LBP-like feature will increase even longer when the local quantization level increases, e.g., if the quantization level is increased to 3 at $(P=8, R=1)$ local area, the length of LBC-like feature will be as long as $10^{3}$ (1000) while the size of LBP-like feature will be $3^{8}$ (6561). Although removing the all-zeros-bins further reduces the size of LBC-like feature, the size of the feature vector still enlarges too much when the local quantization level increases. Hence, an optimal local quantization level should be selected by considering both the additional discriminative information and the computation complexity. Indeed, the local quantization level should not be very high. There are two main reasons.

Firstly, the illumination and gray scale change a lot in the real texture image, that means the gray levels are discontinuous and uneven on the whole image. But on the microcosmic local neighbor area, the changes of the gray value are approximate continuity and uniformity. Thus the gray value differences of the local neighbor pixels are very small. Note that the local acute gradient transformations often occur at the sharp edge area, such as the edge of the foreground and the background. But most of the local gradient transformations are relatively flat and mild at the texture area. To validate this opinion, we have calculated the average local gray value differences of over 5000 natural texture images (with the gray value from 0 to 255), the average local gray value difference is 10.04 and over 90 percent images have the average local gray value difference less than 20. Since the differences of local gray-levels are small, the quantization level has no need to be very high. That is also the potential reason why the local threshold is very small in the experiment of the LTP.

Secondly, the neighbor pixels are finite and the feature histogram will be sparser if the 
quantization level is too high. The LQC histogram is a statistical feature. When the statistical quantities (total pixels) are comparatively less, the statistical error will be relatively higher since the statistical histogram feature is too long and sparse. Especially when the resolution of texture image is very small and the total number of the pixels is very less, the quantization level should be selected even smaller. In this paper, the optimal quantization level of the neighbor pixels is set as 4 according to the experimental performance.

\section{Experiments and discussions}

To evaluate the effectiveness of the proposed method, we carried out a series of experiments on several large and representative databases: the Outex TC10 dataset [36], the XU High Resolution database [21], the UIUC database [22], the Columbia-Utrecht Reflection and Texture (CUReT) database [23], and the KTH-TIPS database [35].

\subsection{Other methods for performance comparisons}

The LTP [12], which can be regarded as another way to increase the local quantization level, is used as a comparison. The CLBP [13], achieves almost the best performance among the single-scale variants of LBP on many representative texture databases, is used as another comparison. As an local counting based method, the proposed LQC is also compared with the CLBC [18]. In addition, the rotation invariant uniform mode LBP riu2 is used in these variants of LBP since the $\mathrm{LBP}^{\text {riu2 }}$ mode performs better than $\mathrm{LBP}^{\mathrm{ri}}, \mathrm{LBP}^{\mathrm{u} 2}$, and normal LBP for rotation invariant texture classification scenario [18].

In the experiments we evaluate different central quantization levels and neighbor quantization levels. We use "LQC_C $(x) \mathrm{N}(y)$ " to denote that the central pixels are quantized 
into $x$ levels and the local neighbor pixels are quantized into $y$ levels, respectively.

\subsection{Dissimilarity measure and multi-scale framework}

In practical applications, several measures have been proposed for discriminating the dissimilarity between two histograms. In this paper, we utilized the $\chi^{2}$ statistics to address the problem. The $\chi^{2}$ statistics is a bin-by-bin distance, which means only the pairs of bins that have the same index are matched. If $H=\left\{h_{i}\right\}$ and $K=\left\{k_{i}\right\}(i=1,2 \ldots B)$ denote two histograms, then $\chi^{2}$ statistics can be calculated as follows:

$$
d_{\chi^{2}}(H, K)=\sum_{i=1}^{B} \frac{\left(h_{i}-k_{i}\right)^{2}}{h_{i}+k_{i}}
$$

In [9],[13], a simple multi-resolution framework is used to improve the classification accuracy, that is, by measuring the dissimilarity as the sum of chi-square distances from all operators of various radiuses. In this paper, assuming that all the methods used the nearest neighborhood classifier for classification. The source codes for the proposed method can be downloaded from the website: http://zycv.890m.com/zyProjectPages/LQCH.zip

\subsection{Experimental Results on Outex TC10 Dataset}

When conducting the experiments on the Outex database, we used the Outex test suits Outex_TC_0010 (TC10), which contains 24 classes of texture images captured under nine rotation angles $\left(0^{\circ}, 5^{\circ}, 10^{\circ}, 15^{\circ}, 30^{\circ}, 45^{\circ}, 60^{\circ}, 75^{\circ}\right.$, and $\left.90^{\circ}\right)$. There are twenty $128 \times 128$ images for each rotation angle and the $24 \times 20$ images of rotation angle $0^{\circ}$ were adopted as the training data. The $24 \times 160$ images of other 8 rotation angles are used for test.

Fig.6 and Fig.7 illustrate the classification rate on TC10 dataset with different central quantization levels and neighbor quantization levels. The neighbor quantization level of the 
LQC is fixed as 4 for testing various central quantization levels in Fig.6, and the central quantization level is set as 6 for testing different neighbor quantization levels in Fig.7. From Fig.6 we can find that the LQC performs better than the CLBP and the CLBC when the neighbor quantization level is set as 4, and the LQC with the central quantization levels of 6 and 7 achieves better results than that of other levels. That demonstrates not always the higher quantization levels obtain the better classification rates. Fig.7 also shows that the LQC with appropriate neighbor quantization levels can obtain better results, but too-high neighbor quantization levels cause the reduction of the discriminative capability. Note that the best neighbor quantization level for Outex TC10 dataset is 4. Moreover, classification rates of different methods on Outex TC10 dataset are listed in Table 1. It can be found that the LQC_C(6)N(4) performs the best among these LBP-like operators.

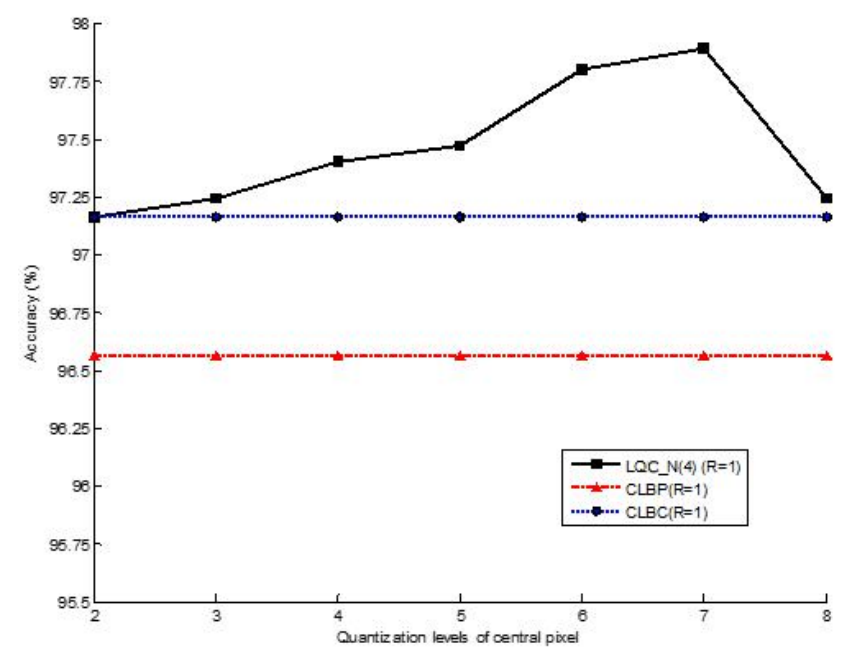

Fig.6. Classification rate on Outex TC10 dataset with different central quantization levels of the proposed LQC. 


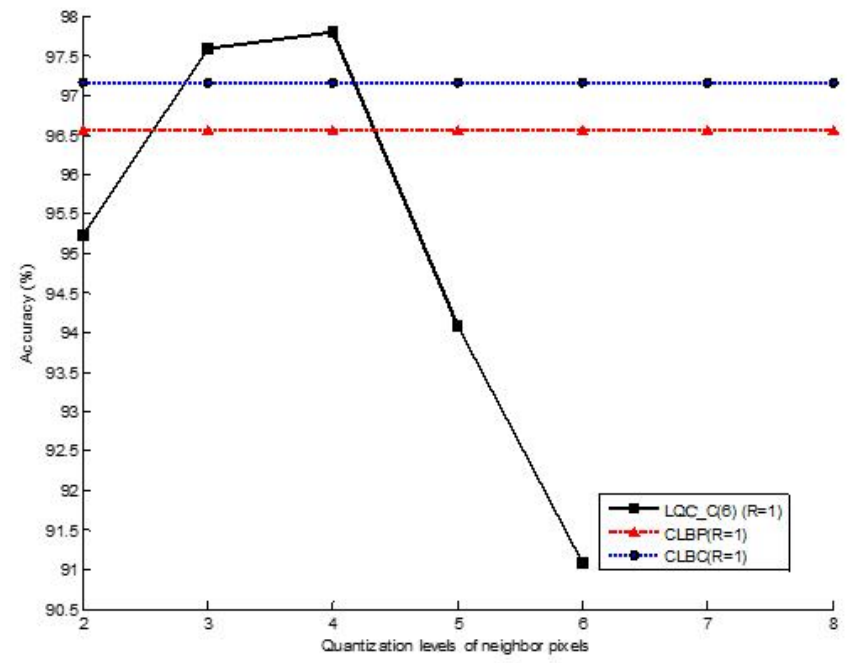

Fig.7. Classification rate on Outex TC10 dataset with different neighbor quantization levels of the proposed LQC.

Table 1. The classification rates $(\%)$ on Outex TC10 dataset

\begin{tabular}{lccc}
\hline & $\mathbf{R}=\mathbf{1 , P}=\mathbf{8}$ & $\mathbf{R = 2 , P = 1 6}$ & $\mathbf{R = 3 , P = 2 4}$ \\
\hline LBP & 84.81 & 89.40 & 95.07 \\
\hline LTP & 93.39 & 96.20 & 97.71 \\
\hline CLBP & 96.56 & 98.72 & 98.93 \\
\hline CLBC & 97.16 & 98.54 & 98.78 \\
\hline LQC_C(2)N(4) & 97.16 & 98.46 & 98.54 \\
\hline LQC_C(6)N(4) & $\mathbf{9 7 . 8 0}$ & $\mathbf{9 8 . 8 0}$ & $\mathbf{9 8 . 9 6}$ \\
\hline
\end{tabular}

\subsection{Experimental Results on XU High Resolution Database}



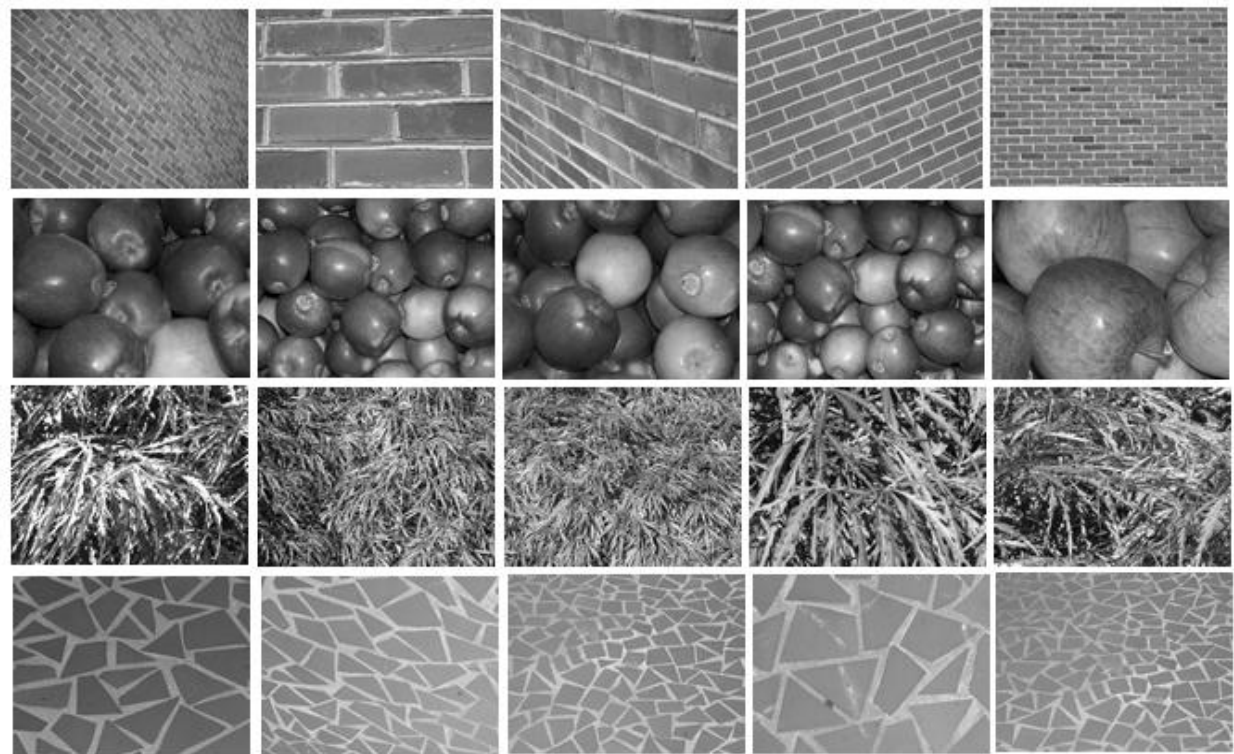

Fig.8. Viewpoint change a lot in XU High Resolution database. Rows 1-4 show images of

four different classes of the XU High Resolution database

The XU High Resolution texture database includes 25 classes and 40 images in each class.

The resolution of each image is $1280 \times 960$. As illustrated in Fig. 8, the images in this database are with very large viewpoint variations. To assess classification performance, $N$ training images are randomly chosen from each class while the remaining 40- $N$ images are used as the test set. The average accuracy over 100 randomly splits are listed in Table 2.

Table 2. The classification rates (\%) on XU HR Databas

\begin{tabular}{ccccccccccccc}
\hline & \multicolumn{9}{c}{$\mathrm{R}=1, \mathrm{P}=8$} & \multicolumn{3}{c}{$\mathrm{R}=2, \mathrm{P}=16$} \\
& 20 & 15 & 10 & 5 & 20 & 15 & 10 & 5 & 20 & 15 & 10 & 5 \\
\hline CLBP & 96.50 & 95.74 & 94.69 & 91.35 & 97.34 & 96.73 & 95.38 & 91.10 & 97.73 & 96.65 & 95.17 & 91.59 \\
\hline CLBC & 96.90 & 96.14 & 94.98 & 91.19 & 97.55 & 96.76 & 95.66 & 91.52 & 97.85 & 96.86 & 95.65 & 91.71 \\
\hline LTP & 87.05 & 85.21 & 80.94 & 71.99 & 91.82 & 90.26 & 87.18 & 79.86 & 93.59 & 92.45 & 89.01 & 80.90 \\
\hline LQC_C(2)N(3) & 95.21 & 94.13 & 92.70 & 88.32 & 94.05 & 93.12 & 90.13 & 83.53 & 93.63 & 92.54 & 90.23 & 85.27 \\
\hline LQC_C(6)N(3) & 96.53 & 95.74 & 94.63 & 91.51 & 95.75 & 94.51 & 92.94 & 89.27 & 95.89 & 94.88 & 93.47 & 90.13 \\
\hline LQC_C(2)N(4) & 97.37 & 96.26 & 94.74 & 91.59 & 98.07 & 97.00 & 95.53 & 92.36 & 98.15 & 97.09 & 95.62 & 92.18 \\
\hline LQC_C(6)N(4) & 97.90 & 97.48 & 96.23 & 93.35 & 98.75 & 97.88 & 96.61 & 93.96 & 98.70 & 98.10 & 97.13 & 94.04 \\
\hline LQC_C(6)N(5) & $\mathbf{9 8 . 1 2}$ & $\mathbf{9 7 . 5 4}$ & $\mathbf{9 6 . 3 9}$ & $\mathbf{9 3 . 6 9}$ & $\mathbf{9 8 . 8 8}$ & $\mathbf{9 8 . 0 0}$ & $\mathbf{9 7 . 0 1}$ & $\mathbf{9 4 . 0 2}$ & $\mathbf{9 8 . 7 4}$ & $\mathbf{9 8 . 2 2}$ & $\mathbf{9 7 . 2 6}$ & $\mathbf{9 4 . 2 3}$ \\
\hline
\end{tabular}

We could get the following findings from Table 2. Firstly, when the local quantization level 
is 3, the LQC_C(2)N(3) and LQC_C(6)N(3) performs better than the LTP on texture images. That is mainly because the LTP is designed for the face recognition problem, and it is no longer strictly invariant to monotonic gray scale transformation since a fixed artificial threshold is used. Secondly, compared LQC_C(2)N(3) with LQC_C(6)N(3), we can find that increasing the quantization level of the central pixel can also enhance the discriminative capability. It should be noted that although the LQC_C(6)N(3) outperforms LQC_C(2)N(3), its feature length is almost three times longer than the LQC_C(2)N(3). Thirdly, when the local quantization level increases to 4, LQC_C(2)N(4) and LQC_C(6)N(4) can achieve higher classification rates than the CLBP and the CLBC. As aforementioned, the length of the LQC feature size also increases a lot when the local quantization level increases. Lastly, LQC_C(6)N(5) merely slightly outperforms LQC_C(6)N(4) by increasing the local quantization level from 4 to 5, but the feature size of LQC_C(6)N(5) is much longer than LQC_C(6)N(4). Thus, the optimal local quantization level is 4 on $\mathrm{XU}$ database by considering both classification rates and the length of feature vector.

By applying the multi-scale scheme, some better results could be obtained. The multi-scale $\operatorname{CLBP}_{(\mathrm{R}=1,2,3)}$ can reach the classification rates of $97.89 \%, 96.89 \%, 95.83 \%$ and $91.96 \%$ for 20 , 15,10 and 5 training samples, respectively. The multi-scale $\operatorname{CLBC}_{(\mathrm{R}=1,2,3)}$ can reach the ones of $98.02 \%, 96.98 \%, 95.49 \%$ and $91.85 \%$ for $20,15,10$ and 5 training samples, respectively. While the proposed multi-scale LQC_C $(6) \mathrm{N}(4)_{(\mathrm{R}=1,2,3)}$ achieves $98.70 \%, 98.10 \%, 97.35 \%$ and $94.74 \%$ for $20,15,10$ and 5 training samples, respectively. Hence, the multi-scale LQC performs better than the multi-scale CLBP and multi-scale CLBC.

\subsection{Experimental results on UIUC database}




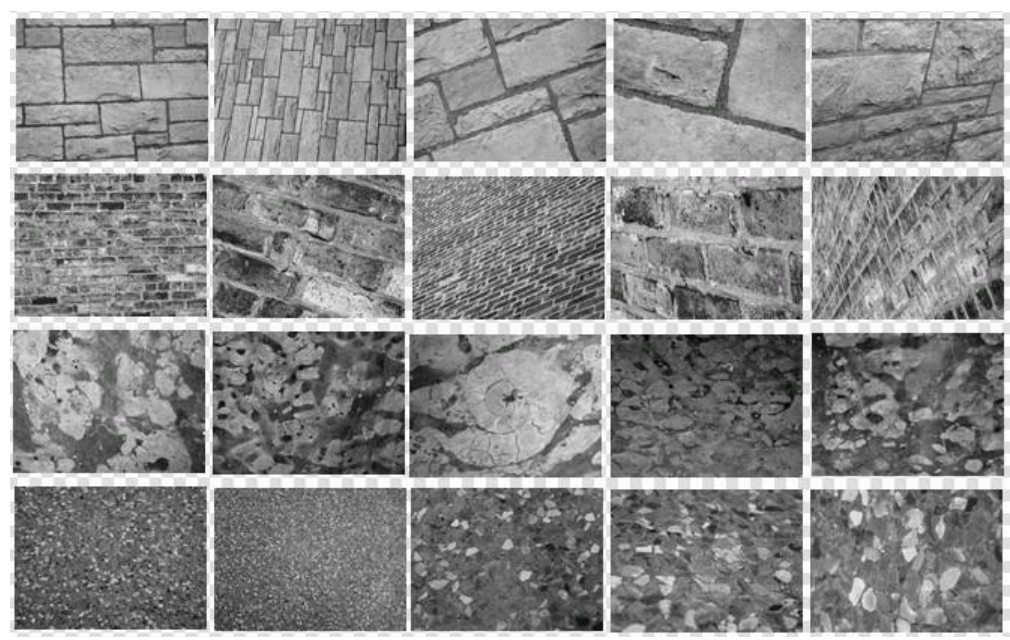

Fig. 9. Viewpoint change a lot in UIUC database. Rows 1-4 show images of four different classes of the UIUC database.

The UIUC texture database includes 25 classes and 40 images in each class. The resolution of each image is $640 \times 480$. As shown in Fig. 9, the database contains materials imaged under significant viewpoint variations. In our experiment, $N$ training images are randomly chosen from each class while the remaining $40-N$ images are used as the test set. The average accuracy over 100 randomly splits are listed in Table 3.

Table 3. The classification rates (\%) on UIUC Database

\begin{tabular}{ccccccccccccc}
\hline & \multicolumn{4}{c}{$\mathrm{R}=1, \mathrm{P}=8$} & \multicolumn{3}{c}{$\mathrm{R}=2, \mathrm{P}=16$} & \multicolumn{3}{c}{$\mathrm{R}=3, \mathrm{P}=24$} \\
\cline { 2 - 13 } & 20 & 15 & 10 & 5 & 20 & 15 & 10 & 5 & 20 & 15 & 10 & 5 \\
\hline CLBP & 87.64 & 85.70 & 82.65 & 75.05 & 91.04 & 89.42 & 86.29 & 78.57 & 91.19 & 89.21 & 85.95 & 78.05 \\
\hline CLBC & 87.83 & 85.66 & 82.35 & 74.57 & 91.04 & 89.66 & 86.63 & 79.48 & 91.39 & 90.10 & 86.45 & 79.75 \\
\hline LTP & 67.16 & 64.29 & 58.20 & 48.15 & 79.25 & 75.80 & 70.77 & 60.34 & 82.34 & 79.10 & 73.94 & 62.19 \\
\hline LQC_C(2)N(3) & 82.94 & 80.85 & 76.61 & 69.95 & 81.80 & 79.68 & 74.75 & 65.85 & 81.64 & 79.32 & 74.76 & 63.22 \\
\hline LQC_C(6)N(3) & 83.79 & 81.67 & 78.47 & 71.58 & 82.22 & 79.94 & 75.90 & 67.61 & 82.54 & 79.45 & 75.43 & 67.13 \\
\hline LQC_C(2)N(4) & 89.45 & 87.70 & 83.58 & 76.23 & 92.32 & 90.58 & 87.67 & 81.48 & 92.94 & 90.39 & 87.54 & 81.64 \\
\hline LQC_C(6)N(4) & $\mathbf{9 0 . 1 2}$ & $\mathbf{8 8 . 5 4}$ & $\mathbf{8 5 . 6 2}$ & $\mathbf{7 8 . 3 8}$ & $\mathbf{9 2 . 6 2}$ & $\mathbf{9 0 . 7 6}$ & $\mathbf{8 7 . 9 7}$ & $\mathbf{8 1 . 9 8}$ & $\mathbf{9 3 . 1 7}$ & $\mathbf{9 0 . 9 1}$ & $\mathbf{8 8 . 1 3}$ & $\mathbf{8 1 . 7 7}$ \\
\hline
\end{tabular}

Similar findings to those in Section 4.3 can be found in Table 3. Firstly, when the local quantization level increases to 4, the LQC_C(2)N(4) and LQC_C(6)N(4) outperforms LQC_C(2)N(3) and LQC_C(6)N(3). Secondly, LQC_C(2)N(4) and LQC_C(6)N(4) still perform better than the CLBP, the LTP and the CLBC. Lastly, the LQC_C(6)N(4) can achieve 
higher classification rates than LQC_C(2)N(4), but its feature sizes are almost three times longer than the LQC_C(2)N(4). Besides, when the local quantization level is as high as 5, the classification rates of the LQC_C(6)N(5) are $93.19 \%, 91.11 \%, 88.34 \%$ and $81.97 \%$ at $(R=3$, $P=24)$ for $20,15,10$ and 5 training samples, respectively. The performance of the LQC_C(6)N(5) is similar to LQC_C(6)N(4), while the feature length of the former one is much longer. Thus, the optimal local quantization level is still 4 on the UIUC database.

By utilizing the multi-scale scheme, the multi-scale $\operatorname{CLBP}_{(\mathrm{R}=1,2,3)}$ can reach the classification rates of $91.57 \%, 89.84 \%, 86.73 \%$ and $78.42 \%$ for $20,15,10$ and 5 training samples, respectively; the multi-scale $\operatorname{CLBC}_{(\mathrm{R}=1,2,3)}$ can reach the ones of $92.42 \%, 90.66 \%$, $87.75 \%$ and $80.22 \%$ for $20,15,10$ and 5 training samples, respectively; the proposed multi-scale LQC_C(6)N(4) $(\mathrm{R}=1,2,3)$ achieves $93.40 \%, 91.68 \%, 89.47 \%$ and $82.05 \%$ for 20,15 , 10 and 5 training samples, respectively. It can be found that the multi-scale LQC still performs better than the multi-scale CLBP and multi-scale CLBC.

\subsection{Experimental results on CUReT database}

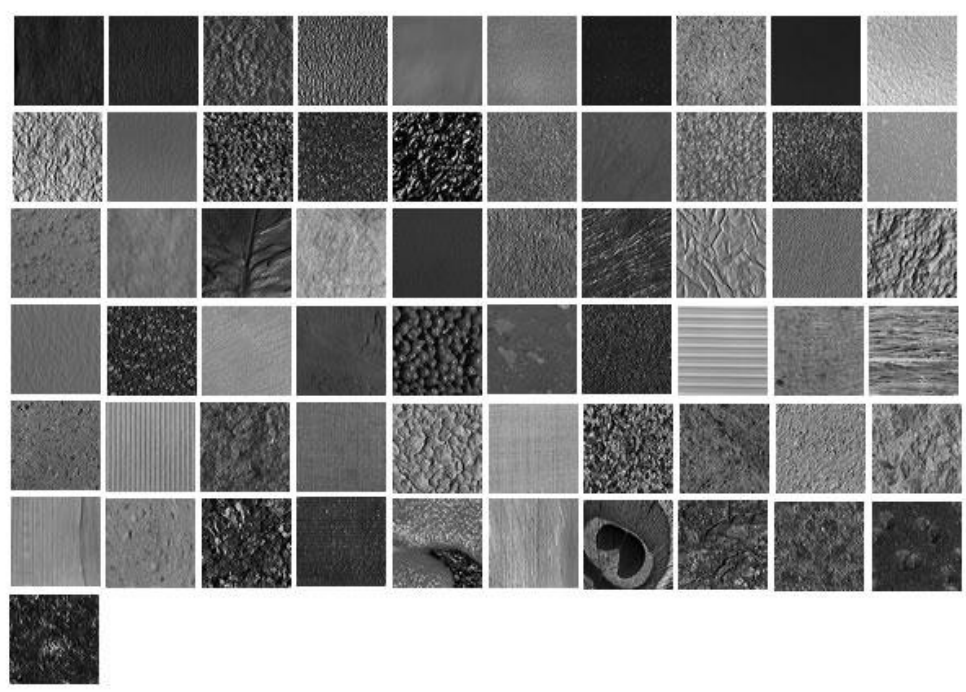

Fig.10. 61 texture images from the CUReT database 
The CUReT database includes 61 classes of textures captured at different viewpoints and illumination orientations (see Fig. 10). In each class, 92 images are selected from the images shot from a viewing angle of less than $60^{\circ}$. As in [13], [24], $N$ images were randomly chosen as training samples from each class. The remaining $92-N$ images were used as test samples. The average classification rates over 100 random splits are listed in Table 43.

We could get the following observation from Table 4 Firstly, the LQC_C(2)N(4) still get better classification results than other variants of LBP. Secondly, different to the performances on the other two high resolution databases, the LQC_C(6)N(4) performs worse than the LQC_C(2)N(4) on CUReT database. The performances of the LQC become worse when the local quantization level further increases. The main reason is that the resolution of the image in CUReT database is very small $(200 \times 200)$. As mentioned before, the statistical error will be increased when the LQC statistical histogram is too long and the pixels are comparatively not enough. As a result, when the texture images are with small resolution, the quantization level should not be set too high. Finally, the optimal local quantization level is still 4 on the CUReT database.

Table 43. The classification rates $(\%)$ on CUReT Database

\begin{tabular}{|c|c|c|c|c|c|c|c|c|c|c|c|c|}
\hline & \multicolumn{4}{|c|}{$\mathrm{R}=1, \mathrm{P}=8$} & \multicolumn{4}{|c|}{$\mathrm{R}=2, \mathrm{P}=16$} & \multicolumn{4}{|c|}{$\mathrm{R}=3, \mathrm{P}=24$} \\
\hline & 46 & 23 & 12 & 6 & 46 & 23 & 12 & 6 & 46 & 23 & 12 & 6 \\
\hline LBP & 80.03 & 73.07 & 67.60 & 58.68 & 84.05 & 79.05 & 72.01 & 62.73 & 86.06 & 81.63 & 75.51 & 67.00 \\
\hline CLBP & 95.19 & 91.20 & 83.81 & 73.44 & 95.35 & 91.24 & 84.66 & 75.41 & 95.38 & 91.77 & 85.01 & 76.16 \\
\hline CLBC & 94.78 & 90.12 & 82.92 & 72.85 & 95.39 & 91.30 & 85.91 & 75.17 & 95.26 & 90.55 & 84.07 & 73.18 \\
\hline LTP & 85.77 & 78.49 & 70.77 & 60.48 & 90.21 & 84.74 & 76.24 & 66.75 & 91.04 & 85.15 & 77.88 & 68.64 \\
\hline LQC_C(2)N(4) & 95.50 & 91.44 & 84.21 & 73.72 & 95.55 & 91.74 & 85.96 & 75.69 & 95.41 & 91.89 & 85.15 & 76.20 \\
\hline LQC_C(6)N(4) & 92.07 & 86.37 & 79.18 & 71.47 & 92.84 & 87.29 & 80.12 & 74.55 & 92.75 & 85.70 & 77.94 & 76.11 \\
\hline
\end{tabular}

4.7 Experimental results on KTH-TIPS database

The KTH-TIPS database contains images of 10 materials. There are eighty one $200 \times 200$ 
images for each material with different combination of pose, illumination and scale. In this paper, 40, 20, 10, and 5 images of each material are utilized for training and the remaining images are used for testing.

The performance of different LBP-like methods on KTH-TIPS is listed in Table 5. From Table 5 we can get the following findings. Firstly, the LTP, the CLBP, and the LQC_C(2)N(4) achieve the highest classification rates at $(\mathrm{R}=2, \mathrm{P}=16)$, while the $\mathrm{LBP}$, the $\mathrm{CLBC}$, and the LQC_C(6)N(4) perform the best at $(\mathrm{R}=1, \mathrm{P}=8)$. Secondly, the proposed LQC can obtain better results than the LTP, the CLBP, and the CLBC. Furthermore, the LQC_C(6)N(4) gets the highest classification rates among these LBP-like operators.

Table 5. The classification rates (\%) on KTH-TIPS Database

\begin{tabular}{|c|c|c|c|c|c|c|c|c|c|c|c|c|}
\hline & \multicolumn{4}{|c|}{$\mathrm{R}=1, \mathrm{P}=8$} & \multicolumn{4}{|c|}{$\mathrm{R}=2, \mathrm{P}=16$} & \multicolumn{4}{|c|}{$\mathrm{R}=3, \mathrm{P}=24$} \\
\hline & 40 & 20 & 10 & 5 & 40 & 20 & 10 & 5 & 40 & 20 & 10 & 5 \\
\hline LBP & 92.85 & 84.59 & 75.10 & 63.69 & 88.32 & 82.02 & 70.59 & 60.54 & 79.83 & 74.87 & 66.10 & 57.29 \\
\hline LTP & 92.37 & 86.08 & 75.34 & 65.13 & 93.24 & 86.20 & 75.73 & 66.84 & 90.39 & 85.79 & 76.01 & 64.59 \\
\hline CLBP & 95.33 & 91.07 & 84.66 & 73.71 & 96.15 & 89.51 & 83.45 & 73.51 & 95.27 & 89.30 & 80.94 & 73.05 \\
\hline CLBC & 95.34 & 90.18 & 83.41 & 72.78 & 93.68 & 88.38 & 79.98 & 71.16 & 91.12 & 85.92 & 74.89 & 68.62 \\
\hline LQC_C(2)N(4) & 95.83 & 91.16 & 84.72 & 74.71 & 96.05 & 90.30 & 82.87 & 73.51 & 94.77 & 89.67 & 82.10 & 71.24 \\
\hline LQC_C(6)N(4) & 96.39 & 91.25 & 84.82 & 74.74 & 96.23 & 90.38 & 83.54 & 74.22 & 95.31 & 89.82 & 82.27 & 74.53 \\
\hline
\end{tabular}

\subsection{Compared with recent non-LBP methods}

The LBP and its variants use specified local patterns to form texture histogram. Instead of utilizing the fixed texture patterns, Verma and Zisserman [30] proposed to cluster the textons by means of the max responses of several filters (VZ_MR8). In [29], they proposed to find a dominant orientation of the local patch to address the rotation invariant issue (VZ_Joint). In these aforementioned works, the spatial information of how pixels are distributed is lost. Aiming at this demerit of the global texture descriptors, Xu et al. [31],[32] proposed a scale invariant texture feature by using the multi-fractal spectrum (MFS). Recently, Xu et al. [33] 
presented a new descriptor based on multi-fractal analysis in wavelet pyramids of texture images (WMFS). In [34], Hanbay et al. proposed four state-of-the-art rotation invariant gradient features based on HOG and CoHOG, i.e., GDF-HOG, Eig(Hess)-HOG, Eig(Hess)-CoHOG, and GM-CoHOG.

The performance of the proposed LQC at $(\mathrm{R}=3, \mathrm{P}=24)$ is compared with that of these non-LBP methods. The experimental results of MFS and WMFS on UIUC database are from [33], and the results of four gradient-based methods on UIUC database are from [34]. The experimental results on three texture databases are listed in Table 6.

We could get the following findings from Table 6. Firstly, the proposed LQC achieves similar classification rate to VZ_Joint on these databases. It should be noticed that the VZ_Joint requires a texton generation process, which often cost several hours. While the LQC is computationally efficient since it does not require texton generation step. By the same hardware the LQC_C(6)N(4) only takes 65 milliseconds, while VZ_Joint spends about 700 seconds to build a texture histogram for one image. Therefore, this LBP-like operator is much more efficient than VZ_Joint. Secondly, compared the LQC with VZ_MR8, the LQC_C(6)N(4) performs better than VZ_MR8 on Outex database, and the VZ_MR8 performs slightly better on other databases. Similar to VZ_Joint, VZ_MR8 also need to cluster the textons from the training set, and total 38 filters are used in the MR8 filter bank. Compared to VZ_MR8, the proposed LQC is more simple and faster. Thirdly, the MFS is proved to be robust to viewpoint changes [31] and it performs impressively on the UIUC database, which contains materials imaged under significant viewpoint variations. Both the LQC and the MFS are computation efficient, and their performances on the UIUC database are also similar to 
each other. Fourthly, WMFS performs much better than other methods on the UIUC database. It also should be noticed that the scales of the images are normalized before the multi-orientation wavelet pyramid multi-fractal analysis in WMFS [33]. The scale estimation and normalization step can greatly enhance the performance of the WMFS, which is not applied in other methods. Finally, two CoHOG based methods, Eig(Hess)-CoHOG and GM-CoHOG [34], achieve much better results than the proposed LQC on UIUC database. Note that the proposed LQC merely counts the number of pixels on different quantization levels and neglects orientation-related information to ensure the LQC is strictly rotation-invariant. The Eig(Hess)-CoHOG and GM-CoHOG can extract abundant gradient orientation information which is omitted in the proposed LQC. Hence, the proposed LQC can be combined with these CoHOG based features to further enhance the discriminative capability. In one word, the LQC achieves comparable classification rate to other recent non-LBP texture classification methods except the WMFS and CoHOG based features on UIUC database.

Table 6. Classification rates (\%) of different methods on three databases

\begin{tabular}{lccccccccc}
\hline & Outex database & \multicolumn{3}{c}{ CUReT database } & \multicolumn{4}{c}{ UIUC database } \\
\cline { 2 - 9 } & TC10 & 46 & 23 & 12 & 6 & 20 & 15 & 10 & 5 \\
\hline LQC_C(2)N(4)(R=3) & 98.54 & 95.41 & 91.89 & 85.15 & 76.20 & 92.94 & 90.39 & 87.54 & 81.64 \\
LQC_C(6)N(4)(R=3) & $\mathbf{9 8 . 9 6}$ & 92.75 & 85.70 & 77.94 & 76.11 & 93.17 & 90.91 & 88.13 & 81.77 \\
VZ_Joint & 98.51 & 96.51 & 93.42 & 88.22 & 79.14 & 93.27 & 92.00 & 88.39 & 80.87 \\
VZ_MR8 & 94.06 & $\mathbf{9 7 . 8 6}$ & $\mathbf{9 5 . 5 4}$ & $\mathbf{9 1 . 2 8}$ & $\mathbf{8 3 . 4 6}$ & 93.96 & 92.68 & 89.32 & 83.07 \\
MFS+SVM [33] & - & - & - & - & - & 92.74 & 91.38 & 88.36 & 82.24 \\
WMFS+SVM[33] & - & - & - & - & - & $\mathbf{9 8 . 6 0}$ & $\mathbf{9 8 . 0 1}$ & $\mathbf{9 6 . 9 5}$ & $\mathbf{9 3 . 4 2}$ \\
GDF-HOG[34] & - & - & - & - & - & 88.63 & - & - & - \\
Eig(Hess)-HOG[34] & - & - & - & - & - & 85.71 & - & - & - \\
Eig(Hess)-CoHOG[34] & - & - & - & - & - & 96.82 & - & - & - \\
GM-CoHOG[34] & - & - & - & - & - & 98.41 & - & - & - \\
\hline
\end{tabular}

\section{Conclusions}


In this paper, we try to search an optimal local quantization level to describe the rotation invariant local texture by proposing a novel local descriptor of Local Quantization Code (LQC). A LBC-like feature is used in the proposed LQC and the average local gray value difference is adopted to set a series of quantization thresholds. The experiment on several real-captured texture databases demonstrates that the proposed LQC outperforms some LBP-like operators, and also illustrates that a low local quantization level (2-4) can effectively characterize the local gray level distribution.

\section{Acknowledgment}

The authors would like to sincerely thank T. Ojala and Z. Guo for sharing the source codes of the local binary pattern and the completed local binary pattern. This work was partly supported by the grant of National Science Foundation of China 61370115, 61402018, the grant of China Postdoctoral Science Foundation, No. 2014M550016, and Shenzhen Peacock Plan.

\section{References}

[1] L. S. Davis, "Polarograms - a new tool for image texture analysis," Pattern Recognition, vol. 13, no. 3, pp. 219-223, 1981.

[2] J. Duvernoy, "Optical digital processing of directional terrain textures invariant under translation, rotation, and change of scale," Applied Optics, vol. 23, no. 6, pp. 828-837, 1984. 
[3] R. K. Goyal, W. L. Goh, D. P. Mital et al., "Scale and rotation invariant texture analysis based on structural property," Proceedings of the 1995 IEEE International Conference on Industrial Electronics, Control, and Instrumentation, vol. 1- 2, pp. 1290-1294, 1995.

[4] G. Eichmann, and T. Kasparis, "Topologically invariant texture descriptors," Computer Vision Graphics and Image Processing, vol. 41, no. 3, pp. 267-281, Mar, 1988.

[5] R. L. Kashyap, and A. Khotanzad, "A model-based method for rotation invariant texture classification," IEEE Transactions on Pattern Analysis and Machine Intelligence, vol. 8, no. 4, pp. 472-481, Jul, 1986.

[6] F. S. Cohen, Z. G. Fan, and M. A. Patel, "Classification of rotated and scaled textured images using Gaussian Markov random field models," IEEE Transactions on Pattern Analysis and Machine Intelligence, vol. 13, no. 2, pp. 192-202, Feb, 1991.

[7] J. L. Chen, and A. Kundu, "Rotation and gray scale transform invariant texture recognition using hidden Markov model," Icassp-92 - 1992 International Conference on Acoustics, Speech, and Signal Processing, vol. 1-5, pp. C69-C72, 1992.

[8] R. Porter, and N. Canagarajah, "Robust rotation invariant texture classification," 1997 IEEE International Conference on Acoustics, Speech, and Signal Processing, vol. I - V, pp. 3157-3160, 1997.

[9] T. Ojala, M. Pietikainen, and T. Maenpaa, "Multiresolution gray-scale and rotation invariant texture classification with local binary patterns," IEEE Transactions on Pattern Analysis and Machine Intelligence, vol. 24, no. 7, pp. 971-987, Jul, 2002. 
[10] M. Heikkila, M. Pietikainen, and C. Schmid, "Description of interest regions with center-symmetric local binary patterns," Computer Vision, Graphics and Image Processing, Proceedings, vol. 4338, pp. 58-69, 2006.

[11] S. Liao, M. W. K. Law, and A. C. S. Chung, "Dominant local binary patterns for texture classification," IEEE Transactions on Image Processing, vol. 18, no. 5, pp. 1107-1118, May, 2009.

[12] X. Y. Tan, and B. Triggs, "Enhanced local texture feature sets for face recognition under difficult lighting conditions," IEEE Transactions on Image Processing, vol. 19, no. 6, pp. 1635-1650, Jun, 2010.

[13] Z. H. Guo, L. Zhang, and D. Zhang, "A completed modeling of local binary pattern operator for texture classification," IEEE Transactions on Image Processing, vol. 19, no. 6, pp. 1657-1663, Jun, 2010.

[14] F. Khellah, "Texture classification using dominant neighborhood structure," IEEE Transactions on Image Processing, vol. 19, no. 12, pp. 3270-3279, 2011.

[15] G. F. McLean, "Vector quantization for texture classification," IEEE Transactions on Systems, Man, and Cybernetics, vol. 23, no. 3, pp. 637-649, 1993.

[16] R. M. Haralick, "Statistical and structural approaches to texture," Proc. IEEE, vol. 67, pp. 786-804, 1979.

[17] M. Varma, A. Zisserman , " Texture classification: Are filter banks necessary?" Proc.IEEE Conf. Computer Vision and Pattern Recognition, vol.2, pp. 691-698, 2003. 
[18] Y. Zhao, D. S. Huang, W. Jia, "Completed Local Binary Count for rotation invariant texture classification," IEEE Transaction on Image Processing, vol.21, pp. 4492-4497, 2012.

[19] J. Zhang, J. Liang, H. Zhao, "Local energy pattern for texture classification using self-adaptive quantization thresholds," IEEE transaction on Image Processing, vol.22, no. 1, pp. 31-42, 2012.

[20] Z. Li, G. Liu, Y. Yang, J. You, "Scale and rotation invariant local binary pattern using scale-adaptive texton and subuniform-based circular shift," IEEE transaction on Image Processing, vol.21, no. 4, pp. 2130-2140, 2012.

[21] http://www.cfar.umd.edu/ fer/High-resolution-data-base/hr_database.htm.

[22] S. Lazebnik, C. Schmid, and J. Ponce, "A sparse texture representation using local affine regions," IEEE Transactions on Pattern Analysis and Machine Intelligence, vol. 27, no. 8, pp. 1265-1278, Aug, 2005.

[23] K. J. Dana, B. Van Ginneken, S. K. Nayar et al., "Reflectance and texture of real-world surfaces," ACM Transactions on Graphics, vol. 18, no. 1, pp. 1-34, Jan, 1999.

[24] S. Lazebnik, C. Schmid, and J. Ponce, "A sparse texture representation using local affine regions," IEEE Transactions on Pattern Analysis and Machine Intelligence, vol. 27, no. 8, pp. 1265-1278, Aug, 2005.

[25] T. Ahonen, et al, "Image description using joint distribution of filter bank responses," Pattern Recognition Letters, vol. 30, no. 4, pp. 368-376, 2009. 
[26] H. Lategahn, S. Gross, et al, "Texture classification by modeling joint distributions of local patterns with Gaussian mixtures," IEEE Transactions on Image Processing, vol. 19, no. 6, pp. 1548-1557, Jun, 2010.

[27] D. Huang, C. Shan, et al, "Local Binary Patterns and Its Application to Facial Image Analysis: A Survey," IEEE Transactions on Systems, Man, and Cybernetics, Part C: Applications and Reviews, vol.41, no.6, pp.765-781, Nov, 2011.

[28] L. Liu, P.W. Fieguth, “Texture Classification from Random Features,” IEEE Transactions on Pattern Analysis and Machine Intelligence, ,vol.34, no.3, pp.574,586, 2012.

[29] M. Varma, and A. Zisserman, "A statistical approach to material classification using image patch exemplars," IEEE Transactions on Pattern Analysis and Machine Intelligence, vol. 31, no. 11, pp. 2032-2047, Nov, 2009.

[30] M. Varma, and A. Zisserman, "A statistical approach to texture classification from single images,” International Journal of Computer Vision, vol. 62, no. 1-2, pp. 61-81, 2005.

[31] Y. Xu, H. Ji, and C. Fermuller, "A projective invariant for texture," International Conference on Computer Vision and Pattern Recognition, pp. 1932-1939, 2006.

[32] Y. Xu, H. Ji, and C. Fermuller, “Viewpoint invariant texture description using fractal analysis," International Journal of Computer Vision, vol. 83, no. 1, pp. 85-100, 2009.

[33] Y. Xu, X. Yang, H. Lin, and H. Ji, “A new texure descriptor using multifractal analysis in multi-orientation wavelet pyramid," International Conference on Computer Vision and Pattern Recognition, pp. 161-168, 2010. 
[34] K. Hanbay, N. Alpaslan, M. F. Talu, et al., "Continuous rotation invariant features for gradient-based texture classification," Computer Vision and Image Understanding, vol. 132, pp. 87-101, 2015.

[35] E. Hayman, B. Caputo, M. Fritz, et al., "On the significance of real-world conditions for material classification," Computer Vision-ECCV, 2004, pp.253-266.

[36] T. Ojala, T. Maenpaa, M. Pietikainen et al., "Outex - new framework for empirical evaluation of texture analysis algorithms," 16th International Conference on Pattern Recognition, vol. I, Proceedings, pp. 701-706, 2002. 